\title{
Optimizing the EU-DEMO pellet fuelling scheme
}

\author{
Peter Thomas Lang ${ }^{\mathrm{a}, *}$, Fabio Cismondi ${ }^{\mathrm{b}}$, Christian Day ${ }^{\mathrm{c}}$, Emiliano Fable ${ }^{\mathrm{a}}$, Antonio Frattolillo ${ }^{\mathrm{d}}$, \\ Curt Gliss $^{\mathrm{b}}$, Filip Janky ${ }^{\mathrm{a}}$, Bernard Pégouriée ${ }^{\mathrm{e}}$ Bernhard Ploeckl ${ }^{\mathrm{a}}$
}

${ }^{a}$ Max-Planck-Insitut für Plasmaphysik, Boltzmannstr. 2, 85748 Garching, Germany

${ }^{\mathrm{b}}$ EUROfusion Consortium, PPPT, 85748 Garching, Germany

${ }^{\mathrm{c}}$ Karlsruhe Institute of Technology (KIT), Karlsruhe, Germany

${ }^{\mathrm{d}}$ ENEA, C.R. Frascati, Via E. Fermi 45, 00044 Frascati (Rome), Italy

${ }^{\mathrm{e}}$ CEA, IRFM, 13108 Saint-Paul-lez-Durance Cedex, France

\section{A R T I C L E I N F O}

\section{Keywords:}

EU-DEMO

Fuelling

Design integration

Pellet

\begin{abstract}
A B S T R A C T
Efficient fuelling will be an essential task in the EU-DEMO. The basic requirement here is to establish the target plasma core density with a minimum particle flux by injecting mm-size solid fuel pellets. Modelling showed this requires a pellet launch from the vessel inboard. Optimization can be achieved by the pellet parameters and the injection geometry; the latter however taking into account boundary conditions resulting from system integration needs. Design activities integrating the pellet transfer system into the vessel and the breeding blanket unveiled several possible variants requiring different levels of technical efforts. Basically, all extra efforts bear the benefit of a deeper and hence more favourable particle deposition. To quantify the potential gain, a full closed loop modelling was performed calculating the required pellet particle flux for any solution considered. Results allow now to balance potential advantages against related efforts required. Furthermore, the analysis tools developed can be employed for even more refined optimization of the pellet actuator tool by e.g. taking into account the interplay of pellet fuelling with burn control requirements.
\end{abstract}

\section{Introduction}

Already the initial considerations on a matter injection system capable to fulfil the requirements on core particle fuelling for the EUDEMO device unveiled the injection of pellets as sole technically feasible option. Pellets, mm-sized bodies formed from solid fuel, must be injected from the torus vessel inboard side to achieve sufficient penetration into the plasma and hence a reasonable fuelling performance [1]. Under steady state conditions, the particle flux leaving the plasma is equal to the time averaged pellet particle flux $\Gamma_{P}$ applied for fuelling. Since these fuelling particles arrive with a virtually nil thermal energy inside the plasma, they result in a convective loss power of

$P_{\text {loss }}=3 \Gamma_{P} k_{B}\langle T\rangle$

with $\langle T\rangle$ the averaged particle temperature. Evidently, this loss power likely causes a reduction of the plasma energy contend resulting in less energy confinement and hence fusion performance. Consequently, optimization of the pellet fuelling system performance aims to minimize $P_{\text {loss }}$ for the envisaged scenario applying a pellet sequence able to establish finally the requested central density $n_{0}$. Besides the parameter of the target plasma, pellet parameters like mass $m_{P}$, repetition rate $f_{P}$, injection speed $v_{P}$ and geometry must be considered for optimization purposes. However, pellet parameters cannot be chosen unconditional, they are linked up by relations like $\Gamma_{P}=m_{P} \times f_{P}$, correlations between the maximum achievable $v_{P}$ and the injection geometry and constrained by restrictions as e.g. limitations on $m_{P}$ to avoid excessive plasma cooling or unbearable local perturbations. Finally, all components of the pellet system have to be integrated into the aggregated construction design of the entire EU-DEMO device factoring in all relevant operational, economical and safety issues.

As a consequence, this optimization in search for the optimal solution has to be conducted as staged and iterative process. The optimal solution is the configuration allowing to establish the required plasma core density with a minimum pellet flux while suiting all the boundary conditions imposed from integration needs. Starting from reasonable assumptions, the basic layout of possible and feasible solutions was sounded out. Once the most eligible approach - the inboard injection - was identified, a refined design was elaborated considering several different options requiring a disparate level of technical efforts. For the most promising variants, detailed modelling of the fuelling behaviour was performed in order to allow for a proper balancing of technical efforts versus expected fuelling performance. Noteworthy, results reported here refer to investigation

\footnotetext{
* Corresponding author.

E-mail address: peter.lang@ipp.mpg.de (P.T. Lang).
} 
performed over a period of more than 5 years within the EU-DEMO plasma reference scenario and the design undergoing a few modest changes. By keeping the according boundary conditions always up-to-date for our investigations, this effects in some minor inconsistency when comparing results from different iterations steps. However, this has apparently no impact on the quality of state-of-the-art solutions considered. Furthermore, available results cannot be regarded final yet. Conventional, they serve as realistic input for the next, even more refined iteration already under way optimizing the fusion burn control [2].

\section{Injection geometry considerations}

\subsection{Basic constraints}

During the initial considerations it was derived $m_{P}=6 \times 10^{21}$ is a suitable choice and hence kept throughout this study. This would correspond e.g. to a cubical deuterium (D) pellet with a side length $l_{P}=4.6 \mathrm{~mm}$ is. Modelling single D pellet injection was performed using the code HPI2, the DEMO1noCD scenario reference plasma was chosen as target plasma [3]. HPI2 is a pellet ablation-deposition code valid for any magnetic and plasma configurations. It computes the pellet ablation taking into account thermal ions and electrons and the suprathermal ions generated by the plasma heating systems; the drift model to calculate the final particle deposition profile is based on the compensation of the cloud polarization by parallel currents [4]. This modelling showed such pellets can yield suitable fuelling impact, provided they are launched from the torus inboard keeping the distance from pellet path intersection point with separatrix with respect to midplane $z_{P} \leq 1.5 \mathrm{~m}$. Furthermore, showing a very drift dominated matter penetration, in EU-DEMO the pellet ablation and particle deposition process will exhibit a characteristics very different with respect to that observed in present day devices [4]. Therefore, optimization of the injection geometry can be conducted by maximizing $v_{P \perp}$, the pellet's speed perpendicular to the separatrix at the intersection point between separatrix and the pellet trajectory. Hence,

$v_{P \perp}=\sin (\alpha) v_{P}$

with $\alpha$ the injection angle between separatrix and designated pellet path (see e.g. Fig. 1).

In case where a curved guiding tube is employed for the pellet transfer to the plasma, a limit on the maximum achievable transfer speed results from centrifugal forces imposing stress on the pellet. The resulting critical pellet speed $v_{c}$ for a tube layout characterised by its minimum bend radius $\mathrm{R}$ was estimated by the "AUG calibrated formula" [1]

$v_{c}=36.4\left[\frac{m}{s}\right] \sqrt{\frac{R}{l_{P}}}$

\subsection{Injection geometry variants}

Integration of the pellet guiding system will be a multi-faceted and complex task, numerous interfaces have been identified. Most important ones yet taken into account are the access into the vacuum vessel and the integration into the breeding blanket (BB). There are different options for a BB under consideration; for this analysis, we picked the design contour of the Helium Cooled Pebble Bed type assuming this one can represent the typical features of any solution and a final adaptation is of minor consequences. For the injection configuration, the basic conventional approach assumes on the pellet transfer at least mostly through guiding system with vessel access from the top in a gap between the toroidal the poloidal field coil. After a presumably straight vertical section through this access position, the subsequent part is formed by a single curved section with radius $R$. At the end of this final section, the pellet leaves the guiding tube approaching the plasma in free flight. Initially, it was assumed the guiding tube can

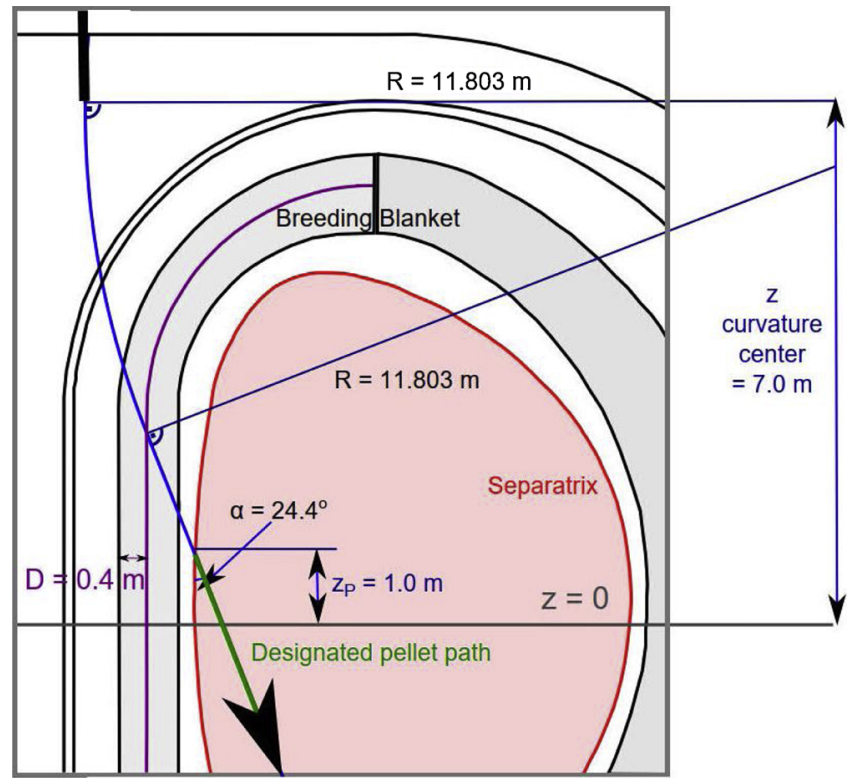

Fig. 1. Example of one possible solution for the D4 variant. Here, the transit position where the curved guiding tube section ends and the straight free flight section begins is determined by the purple contour $\mathrm{D}=0.4 \mathrm{~m}$ inside the outer $\mathrm{BB}$ contour. Both other variants look similar but then the transit position is either the outer BB contour itself (D0) or a contour further inside with $\mathrm{D}=0.6 \mathrm{~m}$ (D6). (For interpretation of the references to colour in this figure legend, the reader is referred to the web version of this article).

penetrate through the entire BB, leaving only a short route to plasma in free flight. Supposed to represent the most appropriate solution for the conventional approach, the geometry optimised with respect to the $v_{P \perp}$ criterion yields $v_{P \perp}=852 \mathrm{~m} / \mathrm{s}$ for a pellet speed of $\mathrm{v}_{\mathrm{P}}=\mathrm{v}_{\mathrm{c}}=1194 \mathrm{~m} / \mathrm{s}$. However, further analysis unveiled this solution has to be disregarded due to excessive heating of the final tube section. Nevertheless, we took this ideal variant as reference to evaluate the potential performance of any other considered injection geometry.

For any acceptable solution the final free flight segment has to start already before or inside the BB. Since there is some angular scatter of the pellet trajectories at the tube exit, some clearance is required for the resulting scattering cone. This has to be taken into account for the $\mathrm{BB}$ design, forcing a considerable cut-out within. In vessel component integration analysis of the fuelling system [5] showed this cut-out has a threefold impact: (i) a reduced tritium breeding rate due to the lost $\mathrm{BB}$ volume, (ii) an increased heating on the vacuum vessel and the coils, (iii) a high neutron flux resulting in an elevated displacement per atom level in the vacuum vessel steel. From the integration analysis, finally three different conventional variants were identified:

- D0 variant - Guiding tube not entering BB at all

- D4 variant - Guiding tube penetrating $0.4 \mathrm{~m}$ into the $\mathrm{BB}$, likely to work with passive cooling

- D6 variant - Guiding tube penetrating $0.6 \mathrm{~m}$ into the $\mathrm{BB}$, requires active cooling

Evidently, the required technical efforts for the guiding tube integration into the BB design increase with increasing penetration. However, in turn the deleterious effects can be lowered by reducing the BB cut-out. For any of the three variants the resulting impact turned out bearable, however just marginally bearable in case of the D0 variant [5].

For all three variants, every geometrically possible solution has been considered employing the CAD tool. Assuming guide tube and injection geometry can be kept within a poloidal plane, these configurations can be covered by a scan of the two parameters. One is the intersection point $\mathrm{z}_{\mathrm{p}}$, the other the $\mathrm{z}$ position of curvature centre of the 
bend section. This becomes clear from Fig. 1, showing the sketch of one single configuration for variant $\mathrm{D} 4$. The radial position of the injection tube is fixed by the boundary condition on the vessel access position; the tube enters vertically. The outer contour of the BB boundary (for D0) respectively the contour of a distinct penetration distance D (for D4 and D6) as well as the separatrix contour (taken for the reference plasma scenario) is fixed. Disregarding scatter effects, the designated pellet trajectory carries on in the direction of the guiding tube at its end. Hence, as indicated in Fig. 1, the free flight line is tangential to the base circle of the curved guide line at the intersection point with the termination contour $(0.4 \mathrm{~m}$ from the outer BB boundary in Fig. 1). All possible reasonable injection geometries are covered by taking into account all injection lines through any separatrix position $\mathrm{z}_{\mathrm{P}}$ and any positive injection angle $\alpha$. Hence, a full injection geometry mapping is granted by variation of the tuple $\left(\mathrm{z}_{\mathrm{P}}, \alpha\right)$. There is a bijective mapping of the configurations isomorphic to the tuple $\left(z_{p}, \alpha\right)$ to those span by the tuple $\left(z_{p}, z\right)$. Hence, the task of finding the best possible configuration for a given variant can be solved by finding the maximum $v_{P \perp}$ under the boundary condition $\mathrm{z}_{\mathrm{p}} \leq 1.5 \mathrm{~m}$ by varying $\mathrm{z}_{\mathrm{p}}$ and $\mathrm{z}$. To do so, for a couple of tuples $\left(z_{p}, z\right)$ spanning the entire relevant configuration regime, resulting $R$ and $\alpha$ values were determined applying CAD. From $R$, $\mathrm{v}_{\mathrm{P}}=\mathrm{v}_{\mathrm{c}}$ was calculated using Eq. (3). Finally, $v_{P \perp}$ was determined from $\mathrm{v}_{\mathrm{P}}$ and $\alpha$. For the example shown in Fig. 1 prescribing $\mathrm{z}_{\mathrm{P}}=1.0 \mathrm{~m}$ and $\mathrm{z}=7.0 \mathrm{~m}$ for the $\mathrm{D} 4$ variant, it yields $\mathrm{R}=11.803 \mathrm{~m}$ and $\alpha=24.4^{\circ}$. From this, $v_{\mathrm{P}}=1844 \mathrm{~m} / \mathrm{s}$ and $v_{P \perp}=762 \mathrm{~m} / \mathrm{s}$ obtained.

Results obtained for all analysed tuples of the D4 variant are shown in Fig. 2, displaying the achievable $v_{P \perp}$ when scanning $\mathrm{z}_{\mathrm{P}}$ for a fixed $\mathrm{z}$ value by a single solid black line (guide to the eye connecting analysed cases represented by the circles); different curves represent different $\mathrm{z}$ values. For reference, the highest performance found when analysing a full BB penetration by the guiding tube is indicated by the horizontal solid grey bar. The best case, $\mathrm{z}_{\mathrm{P}}=1.5 \mathrm{~m}$ for $\mathrm{z}=8.0 \mathrm{~m}$, almost reaching the reference performance. Analysing configurations with yet higher $\mathrm{z}$ values unveiled intersections of the guiding tube with other components, ruling them out as not accessible as indicated in Fig. 2 by the grey shaded area.

However, there is a striking difference between the reference and the best possible D4 solutions. While for the reference the needed $\mathrm{V}_{\mathrm{P}}=1194 \mathrm{~m} / \mathrm{s}$ requirements are still well covered by the available conventional injection technology [6], the best D4 case would require pellets launched at a speed of $1971 \mathrm{~m} / \mathrm{s}$. For such a high pellet speed, at present no reliable launcher technology covering all the needs of a fuelling system is available. Referring to mechanical drivers, at present the speed limit is about $1200 \mathrm{~m} / \mathrm{s}$; for single stage gas gun it is covered up to about $1500 \mathrm{~m} / \mathrm{s}$. There are technologies available to launch pellets up to $3000 \mathrm{~m} / \mathrm{s}$, however not yet matured for application in core particle fuelling in particular due to repetition rates regarded yet inadequate [6]. Hence, all options requiring a pellet launch speed beyond

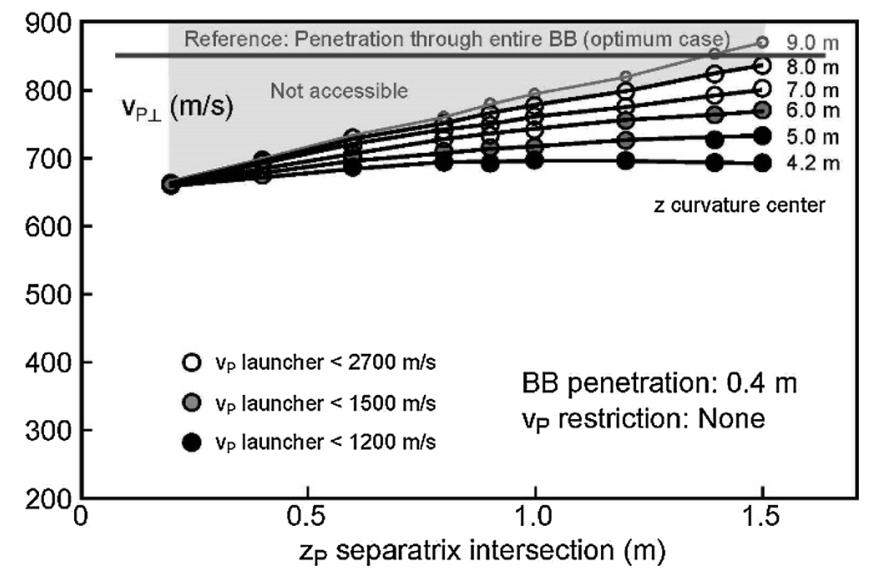

Fig. 2. Resulting perpendicular pellet speed for all analysed solutions for variant D4.

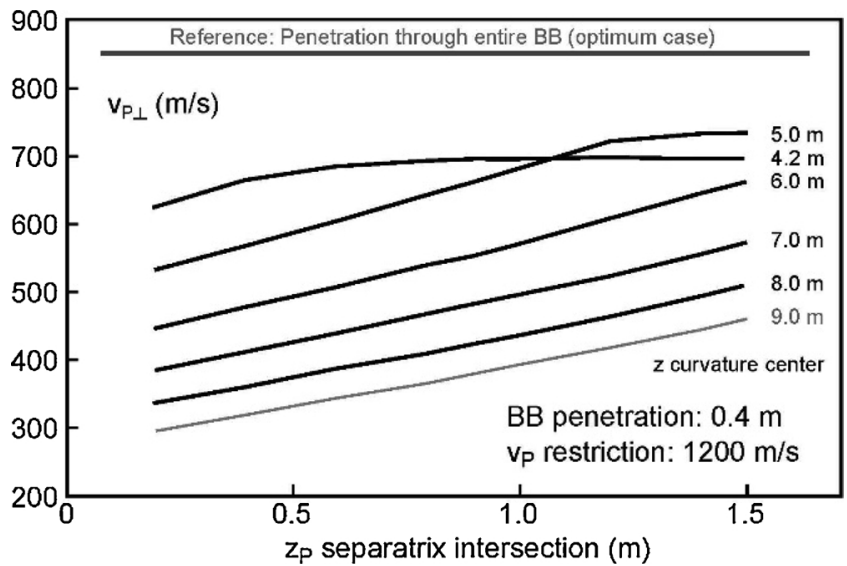

Fig. 3. Resulting perpendicular pellet speed for all analysed solutions for variant D4 taking a limited pellet launch speed of $1200 \mathrm{~m} / \mathrm{s}$ into account.

1500 respectively $1200 \mathrm{~m} / \mathrm{s}$ to date cannot be regarded as technically sound. According solutions are indicated by open circles in Fig. 2, such approaches would either have to rely on a significant progress in pellet launcher technology or would face performance losses. Solutions covered by a technology already at hand are displayed by the filled circles (black: $1200 \mathrm{~m} / \mathrm{s}$, grey $1500 \mathrm{~m} / \mathrm{s}$ ).

To preserve the conservative characteristics of the conventional configurations, thus the technically sound solution of a mechanical launcher device was taken as a basis and a maximum pellet launch speed of $1200 \mathrm{~m} / \mathrm{s}$ set as additional boundary conditions. Recalculation of $v_{P \perp}$ values by setting $\mathrm{v}_{\mathrm{P}}=\max \left\{\mathrm{v}_{\mathrm{c}}, 1200 \mathrm{~mm} / \mathrm{s}\right\}$ considerably modifies the performance, as shown in Fig. 3. For the case shown, now the best solution found is still at the border of the prescribed vertical range with $\mathrm{z}_{\mathrm{P}}=1.5 \mathrm{~m}$ but with a longer straight section down to $\mathrm{z}=5.0 \mathrm{~m}$. The smaller $\mathrm{R}$ now reduces $\mathrm{v}_{\mathrm{c}}$ to $1120 \mathrm{~m} / \mathrm{s}$ and $v_{P \perp}$ to $734 \mathrm{~m} /$ $\mathrm{s}$, quite a bit below the reference values. This case has been selected as best solution representing for the D04 variant as put forward as input for the full fuelling model.

A summary of the best solutions selected this way for the 3 conventional variants is shown in Table 1. Additionally, the table comprises the full $\mathrm{BB}$ penetration reference and one possible solution from the "direct line of sight (DLS)" approach. The data display the key values adopted for the modelling, i.e. the absolute pellet speed, $v_{P \perp}$ and the inclination angle of the injection path with respect to the horizontal plane.

The DLS approach, discussed in detail elsewhere [7], assumes pellets can be injected along direct line of sight trajectories. For such an approach, the speed restrictions resulting from a bend guiding tube would become obsolete with the full speed capability of advanced highspeed launching systems potentially bearing fruit. The according pellet speed assumed for this approach is $3000 \mathrm{~m} / \mathrm{s}$, a velocity which can be achieved by e.g. using multi stage gas guns. To note, pellet rates available for such systems fall still quite short yet with respect to steady fuelling need. Hence, this option would need, beside a sound solution for the injection geometry, significant progress regarding the repetition rate. To compare the possible fuelling performance, for the DLS variant

Table 1

Pellet geometry data for the (meanwhile disregarded) reference scenario and 4 scenarios (3 conventional and one direct line of sight) analysed with the full fuelling model.

\begin{tabular}{llll}
\hline Scenario & $\mathrm{v}_{\mathrm{P}}=\mathrm{v}_{\mathrm{C}}(\mathrm{m} / \mathrm{s})$ & $v_{P \perp}(\mathrm{m} / \mathrm{s})$ & Injection angle $\alpha$ \\
\hline Reference & 1194 & 852 & 45.5 \\
D0 & 1200 & 593 & 29.6 \\
D4 & 1120 & 734 & 40.9 \\
D6 & 1150 & 797 & 43.9 \\
DLS & 3000 & 644 & 12.4 \\
\hline
\end{tabular}


straight injection along an access route regarded doable intersecting the separatrix at $\mathrm{z}_{\mathrm{P}}=1.5 \mathrm{~m}$ was assumed.

\section{Modelling}

\subsection{Approach}

Main goal of the full modelling approach was to provide information for a proper comparison of all the considered injection geometries. Such a comparison requires the knowledge which fuelling flux is needed in order to achieve the requested core density. Hence, the task was to figure out which $f_{P}$ and hence $\Gamma_{P}$ values are needed in any configuration in order to reach the targeted plasma core density. The target plasma chosen for this modelling was EU-DEMO1_2015 [8] with a plasma current $I_{P}=19.6 \mathrm{MA}$ and a central electron density $n_{e}^{0}=1.01 \times 10^{20} \mathrm{~m}^{-3}$. The latter represents a rather high Greenwald fraction of 1.38 , found already ambitious to be achieved in today's tokamak devices [9]. The search for a self-consistent fuelling solution fulfilling such conditions was performed calculating the main plasma parameters by the 1D-radial ASTRA transport code [10]. Assuming a particle confinement time $\tau_{P}=30 \mathrm{~s}$, the sole core fuelling source applied is a repetitive pellet particle source. The fuelling source profile was achieved again from the HPI2 code, inserted into the ASTRA run within a single time step effectuate as instant delta-perturbation.

Since embedding the HPI2 code into the ASTRA run turned out troublesome and very time consuming, a parametrization approach was selected instead found to provide a much faster while still adequately precise solution. In order to allow for broader investigations extending even beyond this study (e.g. to study alternative plasma scenarios or to perform modelling of potential control scenarios) the parametrization was done via deriving a scaling law approximating the pellet particle deposition with a power function depending on seven plasma parameters regarded relevant and $m_{p}$. These plasma parameters are $I_{p}$, the electron temperatures at the separatrix, pedestal top and in the centre, the electron density at the separatrix and the pedestal top and the electron density peaking factor defined at ratio central/pedestal top density. The concluding pellet parameter $\mathrm{v}_{\mathrm{P}}$ was taken as prescribed for any geometry in Table 1. For any of the 4 variants, 200 combinations of the 8 variable parameters were generated by a Monte Carlo approach within a prefixed range and a full HPI2 run performed for every combination. In order to provide an analytic solution of pellet particle deposition profile, it was approximated by a Gaussian distribution characterised by its double variance width $\delta$ and maximum $\lambda$ on the radial grid span by the toroidal magnetic flux $\rho_{\text {tor }}$. For both the maximum and the width a power fit for the 8 variables to the 200 data points generated the scaling laws for each variant. These scaling laws were then employed to calculate the deposition profile for any target plasma within an ASTRA run.

\subsection{Results}

A comparison of the deposition depths as characterised by the maximum and the deposition profile width (values represent twice the variance of the distribution) as calculated by the regression scaling versus the single code run results for the variant D4 are displayed in Fig. 4. Values calculated for each of the 200 single runs by the regression function $\left(\rho_{\text {tor }}=0\right.$ referring to the plasma center while $\rho_{\text {tor }}=1$ represents the separatrix location) are plotted versus values obtained from the HPI2 code (red squares). In both boxes, the solid bisectrix hence reflects the fit function itself.

Already from the centre of mass determined for all the variants it becomes clear there is little difference with respect to fuelling performance expected for the 3 conventional variants while no benefit can be achieved at least for the investigated DLS solution. This can be realised from Table 2, summarizing both the medium deposition depth and the width of the deposition profiles determined for the 200 data calculated
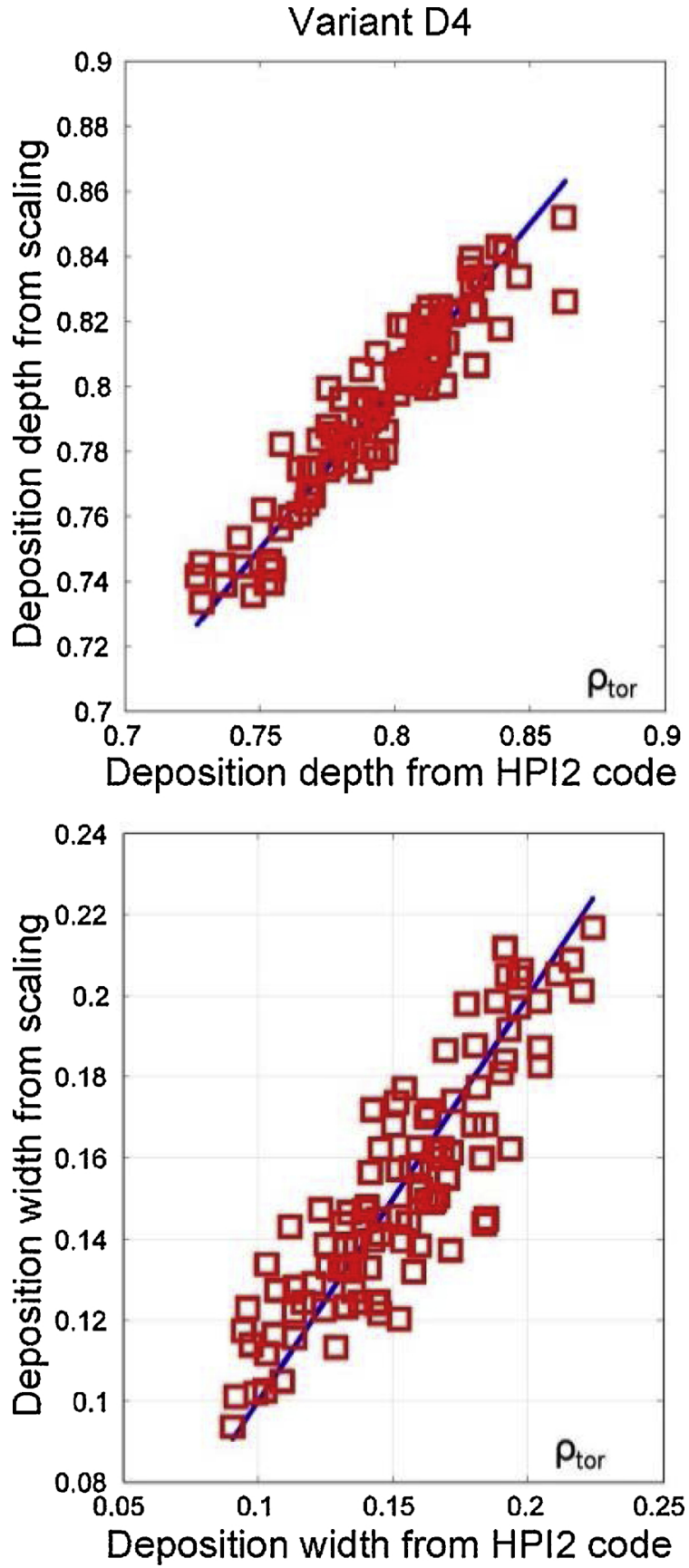

Fig. 4. Maximum (upper) and width (lower) of the pellet particle deposition profile calculated for 200 single full variant D4 HPI2 code runs as calculated by the according scaling versus exact values with respect to the $\rho_{t o r}$ coordinate. The fit function itself hence is represented by the bisectrix (blue solid lines). (For interpretation of the references to colour in this figure legend, the reader is referred to the web version of this article).

Table 2

Mean value of the maximum and the width (double variance) with respect to the $\rho_{\text {tor }}$ coordinate for all 4 considered variants.

\begin{tabular}{lllll}
\hline Variant & D0 & D4 & D6 & DLS \\
\hline$\lambda\left[\rho_{\text {tor }}\right]$ & 0.8078 & 0.7931 & 0.7891 & 0.8398 \\
$\delta\left[\rho_{\text {tor }}\right]$ & 0.1307 & 0.1525 & 0.1298 & 0.0773
\end{tabular}


for each variant.

The full ASTRA code run for all the conventional variants unveiled a $f_{P}$ slightly above $1 \mathrm{~Hz}$ is already sufficient to yield the requested core density. For the according $\Gamma_{P}=7 \times 10^{21} s^{-1}$ hence, due to $\tau_{P}=30 \mathrm{~s}$, the plasma particle inventory $N_{e}=2.1 \times 10^{23}$ within a confined volume of $2500 \mathrm{~m}^{3}$ yields $\left\langle n_{e}\right\rangle=8.3 \times 10^{19} \mathrm{~m}^{-3}$ as requested for the scenario [8].

The estimated flux, one of the main results demanded from this study, can now be used as crucial input for further analysis of the EUDEMO pumping system and fuel cycle. It was also employed already for a first, explorative study on simultaneous density and burn control [2]. From these investigations strong indications were found that, assuming realistic pellet delivery reliabilities (ratio pellets delivered/ pellets requested) of about 0.95 , density variations caused by the low frequency pellet delivery like result in unbearable variations of the fusion burn power. From these initial control considerations, a pellet size reduction to about $1 / 3$ of the size assumed so far seems advisable, resulting in a more frequent but smoother pellet induced swing of the plasma parameter. More detailed investigations are currently under way and will be reported elsewhere [2].

\section{Conclusions and outlook}

The presented study was aiming to elaborate a suitable solution for the core particle fuelling of EU-DEMO. Inboard pellet injection was identified as suitable technique. Besides a conservative conventional schedule, relying only on proven technical potential and a guiding tube based pellet transfer fully compatible with the current plant design, also a variant assuming direct line of sight injection was considered. Several variants for the conservative approach have been worked out, characterised by a potentially improved fuelling performance at the expense of more technical efforts required to integrate the guiding system into the breeding blanket. Recent results applying a tool for a full modelling of the pellet fuelling tool enabled also for a widening the degree of integration. Now, also concerns about feed back controlling plasma performance can be taken into account. Here, it turns out differences between all conventional variants with respect to fuelling behaviour are rather marginal - hence for a final selection aspects like the performance of the breeding blanket or its shielding effects might become most prioritized. Moreover, it appears the initial choice of the pellet mass has to be reconsidered. Lowering to $m_{P} \approx 2 \times 10^{21}$ will likely appease excursion of core plasma parameters to a bearable magnitude. Since the expected fuelling flux will be still moderate, this seems adequate despite smaller pellets will likely result in less penetration and consequently in a mild enhancement of the requested flux. According investigations, aiming to derive an optimized pellet size are under way.

Once this information of an optimized $m_{P}$ value are at hand, the next iteration step on optimizing the injection geometry will be performed.
This will take into account the alteration on $\mathrm{v}_{\mathrm{c}}$ stemming from its mass dependence, employ both an up to date plant design and plasma scenario and extend the analysed $\mathrm{z}_{\mathrm{p}}$ range beyond the current restriction to $1.5 \mathrm{~m}$.

\section{CRediT authorship contribution statement}

Peter Thomas Lang: Conceptualization, Methodology, Writing original draft. Fabio Cismondi: Investigation, Resources. Christian Day: Investigation, Resources. Emiliano Fable: Investigation, Resources, Writing - review \& editing. Antonio Frattolillo: Investigation, Resources. Curt Gliss: Investigation, Resources. Filip Janky: Investigation, Resources. Bernard Pégourié: Investigation, Resources. Bernhard Ploeckl: Investigation, Resources, Writing - review \& editing.

\section{Declaration of Competing Interest}

None.

\section{Acknowledgments}

This work has been carried out within the framework of the EUROfusion Consortium and has received funding from the Euratom research and training programme 2014-2018 and 2019-2020 under grant agreement No 633053. The views and opinions expressed herein do not necessarily reflect those of the European Commission.

\section{References}

[1] P.T. Lang, et al., Considerations on the DEMO pellet fuelling system, Fusion Eng. Des. 96-97 (2015) 123-128.

[2] F. Janky, et al., DEMO Plasma control needs, confirmed invited presentation, SOFT (2020) Publication in preparation.

[3] R. Wenninger, et al., DEMO divertor limitations during and in between ELMs, Nucl. Fusion 54 (2014) 114003.

[4] B. Pégourié, et al., Physical constraints on the design of the DEMO pellet fueling system, Proc. 43th EPS Conference on Controlled Fusion and Plasma Physics, Leuven, 2016 P4.076.

[5] F. Cismondi, et al., Progress in EU-DEMO in-vessel components integration, Fusion Eng. Des. 124 (2017) 562-566.

[6] S.K. Combs, L.R. Baylor, Pellet-injector technology -Brief history and key developments in the last 25 years, Fusion Sci. Technol. 73 (2018) 493-518.

[7] A. Frattolillo, et al., Addressing the feasibility of inboard direct-line injection of high-speed pellets, for core fueling of DEMO, Fusion Eng. Des. (2020) in press.

[8] R. Wenninger, et al., The physics and technology basis entering European system code studies for DEMO, Nucl. Fusion 57 (2017) 016011.

[9] P.T. Lang, et al., Feedback controlled reactor relevant high-density high-confinement scenarios at ASDEX Upgrade, Nucl. Fusion 58 (2018) 036001.

[10] G.V. Pereverzev, Rep. IPP 5/42, Max-Planck-Institut für Plasmaphysik, Garching, 1991. 